\title{
Edad y crecimiento de Chondrostoma toxostoma Vallot, 1837 en el curso inferior del río Ebro (Tarragona - España)
}

\author{
Adolfo de Sostoa y Francisco J. de Sostoa
}

Departamento de Zoología (Vertebrados). Facultad de Biología. Universidad de Barcelona. Barcelona-28.

\begin{abstract}
SUMMARY
AGE AND GROWTH OF Chondrostoma toxostoma VALLOT, 1837 IN THE LOWER COURSE OF THE EBRO RIVER. (TARRAGONA, NE SPAIN).
\end{abstract}

They have been studied 184 exemplares of Chondrostoma toxostoma, captured by several methods in the last $50 \mathrm{~km}$ of the lower strech of Ebro river (Tarragona, NE Spain) during 1980 and 1981. So as to determine the age, the fish scales were utilized. The growth models utilized were those of VON BERTALANFFY (1938); the asymthote calculation was made by the graphic method (FORD 1933; WALFORD 1946); ant the growth rate and " $t_{0}$ " were determined from values of age and length (DICKIE 1978). Equations of ponderal and lineal growth are given, as well as the length-weigth relations, for both sexes.

\section{INTRODUCCION}

En el transcurso de los últimos años se han publicado numerosos trabajos sobre biología de Cyprinidae europeos y más concretamente sobre el género Chondrostoma, en Europa septentrional se han publicado diversos trabajos como los de PRAWOCHENSKI (1963), HOCHMAN \& JIRASEK (1969), KLIMCZYK-JANIKOWSKA (1973), VELEDAR \& KOSORIC (1974), LOSOS et al. (1980). En Europa meridional el número de publicaciones es muy escaso (LUDINI \& NARDI, 1979; LOBON-CERVIA \& ELVIRA, en prensa). De aquí la motivación que nos indujo a estudiar la población de Chondros- toma toxostoma que habita en el tramo final del río Ebro y en los canales principales de riego (canal de la derecha y canal de la izquierda) paralelos a ambos lados del cauce. El área de colonización de esta especie en el río está limitada por la salinización de las aguas en los últimos kilómetros la cual aumenta durante los meses de máximo estiaje.

\section{MATERIAL Y METODOS}

Durante los años 1980-1981, se han capturado en el tramo final del rio Ebro un total de 184 ejemplares de Chondrostoma toxostoma de los cuales 120 eran hembras y 
64 machos. La captura se ha realizado mediante diversos artes de pesca tradicional (salabres de mano, trasmallos, etc.), así como utilizando la pesca eléctrica, con corriente continua rectificada de 100 a $250 \mathrm{~V}$ y alimentada por un generador eléctrico de $1 \mathrm{~kW}$.

A los ejemplares, tras su captura, se les medía la longitud standard (Lst) en milímetros y el peso del individuo sin eviscerar, éste con una precisión de $0,01 \mathrm{gr}$. El sexo de los individuos fue determinado por disección de manera directa cuando se hallan en actividad sexual y mediante técnicas histológicas en la época de reposo sexual. Asimismo, se procedió a la extracción de los huesos operculares $y$ de las escamas.

\section{CALCULO DE LA EDAD}

Para la determinación de la edad de los individuos se ha utilizado la lectura de escamas, las cuales se extraian del flanco izquierdo en la zona mediosuperior por encima de la línea lateral y delante de la aleta dorsal. Posteriormente se procedía a limpiarlas en una solución de $\mathrm{KOH}$ del 2 al $5 \%$ durante 12 horas. Cuatro escamas de cada ejemplar se disponian entre porta y cubreobjetos, y se leian directamente con la ayuda de un proyector de microfilms, provisto de lentes de 10 y 30 aumentos.

Las clases de edad se definen según el número de anillos verdaderos ("annuli") encontrados en las escamas. La aparición de los anillos en las escamas tiene lugar en los meses de mayo y junio. Los individuos que no presentan ningún anillo pertenecen a la clase de edad " 0 ", es decir no han cumplido el primer periodo invernal. Pertenecen a la clase "I" los que presentan un anillo, equivalente como mínimo a un año de vida; cabe señalar que en el caso de especies de reproducción estival, como la que se contempla, el primer anillo verdadero se forma aproximadamente a los 16 meses de vida. Las demás clases de edad corresponden a la aparición sucesiva de anillos.

\section{ECUACIONES DE CRECIMIENTO}

En el presente trabajo se han utilizado los modelos de crecimiento de VON BERTALANFFY (1938), cuyas ecuaciones para el crecimiento son:

$$
\begin{aligned}
& \mathrm{L}_{t}=\mathrm{L}_{\infty}\left[1-\mathrm{e}^{-k(t-t o)}\right] \\
& \text { (crecimiento lineal) } \\
& \mathrm{W}_{t}=\mathrm{W}_{\infty}\left[1-\mathrm{e}^{-k(t-t o)}\right]^{n} \\
& \text { (crecimiento ponderal) }
\end{aligned}
$$

El cálculo de la asíntota del crecimiento lineal $\left(L_{\infty}\right)$ se realiza mediante el método gráfico de FORD-WALFORD (1946), por regresión lineal entre las longitudes medias dc cada edad $\left(\mathrm{L}_{t}\right)$, con las longitudes medias de la edad siguiente $\left(L_{t}+1\right)$, obteniéndose la ecuación de la recta $\mathrm{L}_{t+1}=\mathrm{a}+b \cdot \mathrm{L}_{t}$. La intersección de esta recta con la recta $L_{t}=$ $\mathrm{L}_{t} \quad 1$, sería el punto en el que no habría crecimiento; dicho punto debe satisfacer las dos ecuaciones, es la asíntota, y se ubtiene de la fórmula:

$$
\mathrm{L}_{\infty}=\frac{a}{1-j}
$$

Para el cálculo de la tasa de crecimiento $(k)$ y la edad teórica en que la longitud es cero $(t o)$, se han utilizado todas las longitudes observadas y sus correspondientes edades, mediante una transformación logaritmica de la ecuación de VON BERTALANFFY (DICKIE, 1978) y por regresión lineal con el método de los minimos cuadrados. Otros autores obtienen la tasa de crecimiento directamente de la recta de FORD-WALFORD, ya que la pendiente de dicha recta es $b=$ $\mathrm{e}^{-k}$, pero parece más conveniente deducirla de todos los valores reales y no a partir de las longitudes medias de cada edad.

La asíntota del peso $\left(W_{\infty}\right)$ se calcula a partir de la relación longitud standard-peso, siendo $n$ el exponente de dicha relación.

Para dar una estima del ajuste de las ecuaciones de crecimiento con los valores medios 
reales, utilizaremos la "desviación standard de los logaritmos", (S log $_{\text {log }}$ KRUER, 1973), que se obtiene de:

$$
S_{\log }= \pm \frac{(\log y-\log \bar{y})^{2}}{n-3}
$$

de donde $y=$ valor medido, $\bar{y}=$ valor calculado, y $\mathrm{n}=$ número de pares. Para poder compararla el mismo autor sugiere usar la forma porcentual " $\mathrm{S} \%$ ", obtenida de la fórmula:

$$
S \%=\left(100.10^{S_{\log }}\right)-100
$$

La relación longitud standard-peso viene definida por la ecuación:

$$
W=\mathbf{a} \cdot \text { Lst } \mathbf{b}
$$

siendo $\mathrm{W}$ el peso en gramos y Lst la longitud standard en milimetros. La determinación de las constantes a y b se realiza por una regresión lineal, según el método de los mínimos cuadrados, entre los logaritmos de las longitudes standard y los pesos:

$$
\mathrm{W}=\mathbf{a}+\mathbf{b} . \ln \text { Lst. }
$$

\section{RETROCALCULO}

Para retrocalcular las longitudes de las edades pretéritas se selecciona una escama, entre las montadas, y se procede a medir la longitud de los diferentes anillos, sobre el radio posterior (HOFSTEDE, 1970), a partir del centro de dicha escama. La longitud de dichos radios se mide por proyección sobre una escala transparente y graduada en milimetros de las escamas en una lectora de microfilms, con un aumento de $10 x$.

El retrocálculo se basa en la relación existente entre la longitud de la escama y la longitud del pez. Dicha relación no es direca, en la mayoría de los casos, y por ello se utiliza la ecuación de FRASER-LEE (1920):

$$
\mathrm{L}_{t}=\mathrm{a}+\frac{\mathrm{R}_{t}}{\mathrm{R}_{n}} \cdot\left(\mathrm{L}_{n}-\mathrm{a}\right)
$$

siendo $L_{t}$ la longitud a la edad $t$ que queremos obtener, $\mathrm{R}_{t}$ el radio a la edad $t, \mathrm{R}_{n}$ el radio total de la escama, $L_{n}$ la longitud del ejemplar que corresponde a $R_{n}$ y a un factor de corrección, que se obtiene por una regresión lineal sobre la longitud de la escama como variable independiente y la longitud del ejemplar como variable dependiente:

$$
\mathrm{L}_{n}=c+d . \mathrm{R}_{n}
$$

siendo $c$ la ordenada en el origen.

\section{RESULTADOS}

EDAD

Las edades máximas encontradas son $1 a^{\circ}$ clase VII para las hembras, y la clase V para los machos. La especie estudiada, por su carácter estenohalino-limnético (DEMESTRE et al. 1977), está en el limite de sus posibilidades de colonización en este área; por lo que resulta muy probable que ciertas clases de edad no aparezcan o estén subrepresentadas.

\section{CRECIMIENTO LINEAL}

Los valores calculados para el crecimiento lineal representan en realidad las longitudes medias de cada clase de edad, es decir, la talla más probable que alcanzarían los individuos al aumentar el tamaño de la muestra.

En la figura 1 podemos ver las representaciones gráficas de los modelos gráficos de FORD-WALFORD (1946) utilizados en el cálculo de las asíntotas de las longitudes, cuyas ecuaciones son (fig. 1):

$$
\mathrm{L}_{t+1}=41,234+0.803 . \mathrm{L}_{t}
$$

O'

$$
\mathrm{L}_{\infty}=\frac{41,238}{0,803}=210.04 \mathrm{~mm}
$$




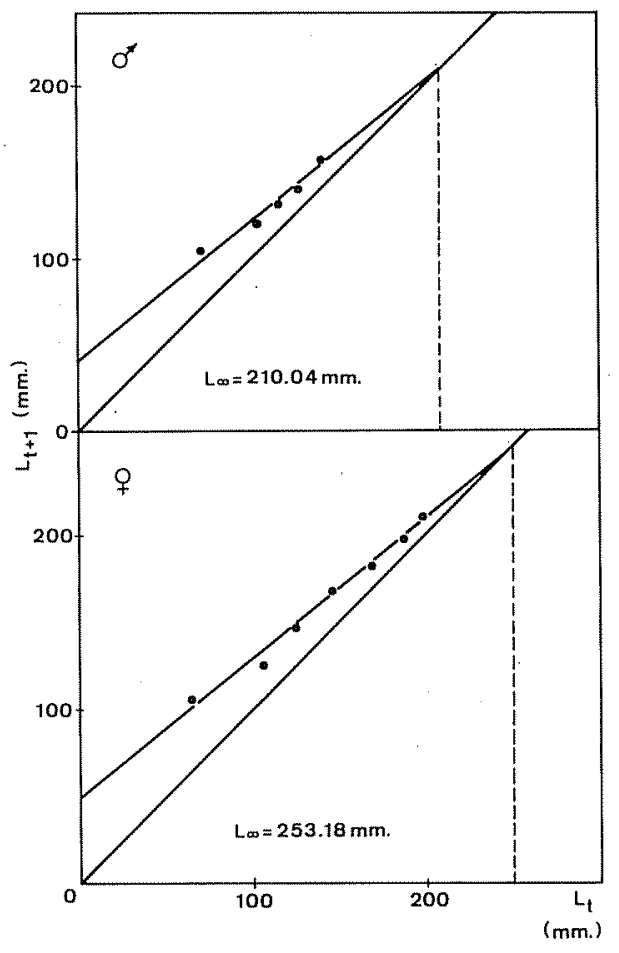

Fig. 1. Fcuaciones de FORD-WALFORD para el cálculo de las asíntotas de ambos sexos de Chondrostoma toxostoma.

$$
\begin{aligned}
& \mathrm{L}_{t+1}=47,248+0.813 \cdot \mathrm{L}_{t} \\
& \quad \mathrm{~L}_{\infty}=\frac{47,248}{0,813}=253,18 \mathrm{~mm} .
\end{aligned}
$$

Las tasas de crecimiento (tasa de catabolismo) $k$, el tiempo de talla cero $t_{\mathrm{o}}$ (que carece de significado biológico) y el coeficiente de anabolismo $\mathrm{E}=k$. Loo son

$\begin{array}{lccc} & t_{\mathbf{o}} & k & \mathrm{E} \\ \text { machos: } & -2,463 & 0,184 & 38,64 \\ \text { hembras: } & -1,670 & 0,193 & 48,86\end{array}$

asi pues las ecuaciones de crecimiento de VON BERTALANFFY (1938) son para nuestros ejemplares:

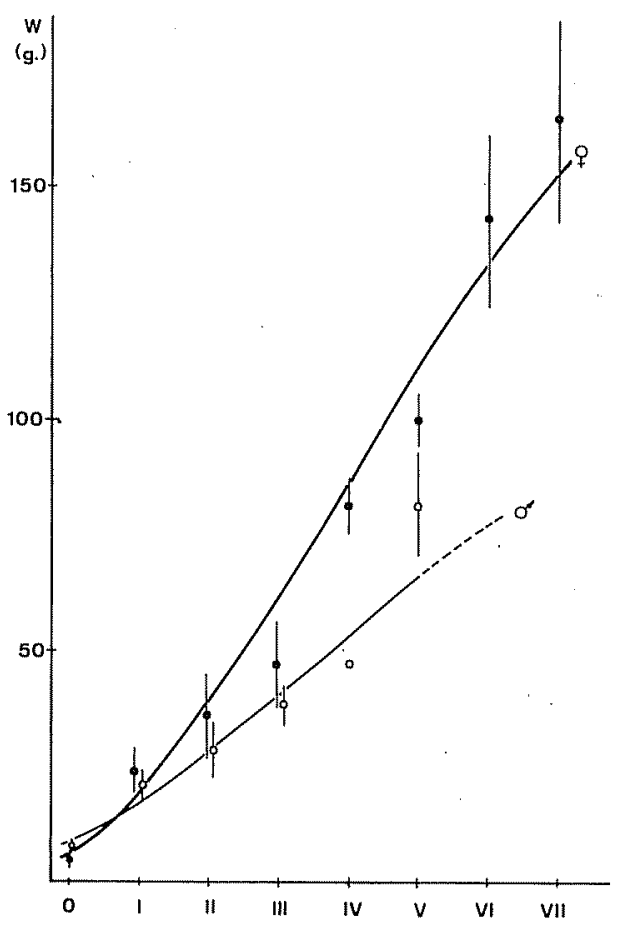

Fig. 2. Representación gráfica de los valores teóricos de longitud (modelo de VON BERT ALANFFY (1938)), los valores medios reales, el intervalo de confianza del $95 \%$ e incrementos anuales en longitud de Chondrostoma toxostoma.

$$
\begin{array}{ll}
: & \mathrm{L}_{t}=210,04\left[1-\mathrm{e}^{-0,184(\mathrm{t}+2,463)}\right] \\
: & \mathrm{L}_{t}=253,18 \cdot\left[1 \cdot-\mathrm{e}^{-0,193(\mathrm{t}+1,670)}\right]
\end{array}
$$

En la figura 2 se observa la representación gráfica de las longitudes de cada clase de edad, asi como los valores de las longitudes medias observadas y los intervalos de confianza del $95 \%$, de los dos sexos por separado. Asimismo se representan los ingresos anuales en longitud standard $(\Delta \mathrm{Lst})$. (Ver también tablas I y II).

Los valores de ajuste de las dos curvas, "S $S_{\text {log" }}$ y "S\%" de KRUGER (1973) son:

$$
\mathrm{S}_{\mathrm{Iog}} \quad \mathrm{S} \%
$$

machos: $\quad 0,031923.7,6276$

hembras: $0,023400 \quad 5,5350$


TABLA I. Valores teóricos de longitud standard (Lst), incrementos anuales en longitud ( $\triangle \mathrm{L} s \mathrm{st})$, pesos (W) y tasas instantáneas de crecimiento $(G)$, para los MACHOS de Chondrostoma toxostoma.

$\begin{array}{lrcrc}\text { Edat } & \text { Lst } & \text { Arst } & W & \text { G } \\ & & & & \\ \text { O } & 76,24 & 22,61 & 8,00 & 0,756 \\ \text { I } & 98,85 & 18,81 & 17,06 & 0,756 \\ \text { II } & 117,66 & 15,63 & 28,32 & 0,363 \\ \text { III } & 133,29 & 13,00 & 40,74 & 0,271 \\ \text { IV } & 146,29 & 10,81 & 53,42 & 0,207 \\ \text { V } & 157,10 & & 65,75 & \end{array}$

TABLA II. Valores teóricos de longitud standard (Lst), incrementos anuales de longitud ( $\triangle \mathrm{Lst}$ ), pesos (W) y tasas instantáneas de crecimiento $(G)$, para las HEMBRAS de Chondrostoma toxostoma.

\begin{tabular}{lrrrc} 
Edad & \multicolumn{1}{c}{ Lst } & ALst & W & $G$ \\
& \multicolumn{4}{c}{ SL } \\
O & 69,86 & & & \\
I & 102,08 & 32,22 & 6,59 & 1,105 \\
II & 128,64 & 25,56 & 19,93 & 0,674 \\
III & 150,52 & 21,88 & 39,11 & 0,458 \\
IV & 168,57 & 18,05 & 61,85 & 0,330 \\
V & 183,44 & 14,87 & 86,03 & 0,246 \\
VI & 195,69 & 12,25 & 110,08 & 0,188 \\
VII & 205,80 & 10,11 & 132,93 & 0,146
\end{tabular}

vemos que las hembras presentan un mejor ajuste que los machos, debido, seguramente a que se dispuso de una muestra mayor. Sin embargo, las desviaciones standard porcentuales " $\mathrm{S} \%$ " de ambos sexos se encuentran en el intervalo del $90 \%$.

\section{RELACION LONGITUD-PESO}

Las ecuaciones obtenidas, asi como los coeficientes de correlación son:

$$
\begin{aligned}
& O^{7}: \ln . W=2,912 . \ln . \text { Lst } \cdots \cdot 10,535 \\
& (\mathrm{r}=0,97) \\
& W=2,658 \cdot 10^{-5} \text {. Lst } 2,912 \\
& \text { Q : } \ln . W=2,915 . \text { In Lst } \cdots 10,493 \\
& (\mathrm{r}=0, \overline{98}) \\
& W=2,771 \cdot 10^{-5} \text {. Lst } 2,915
\end{aligned}
$$

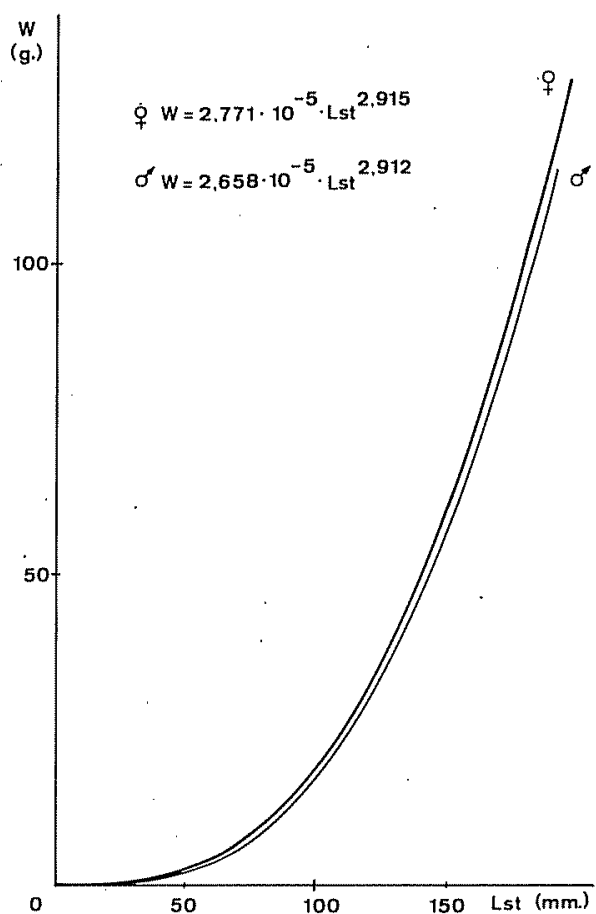

Fig. 3. Relación longitud standard-peso de ambos sexos de Chondrostoma toxostoma del río Ebro.

En la figura 3 se representan los valores de dichas relaciones.

\section{CRECIMIENTO PONDERAL}

Al igual que en el crecimiento lineal, los valores calculados corresponden a los pesos medios para cada clase de edad. Las asintotas para el crecimiento ponderal se han calculado a partir de las relaciones longitud standard-peso para cada sexo (fig. 4), y no de un ajuste funcional a nivel poblacional, asi tenemos:

$$
\begin{aligned}
& \sigma^{\prime}: W_{\infty}=2,658 \cdot 10^{-5} \cdot 210,04^{2,912}= \\
& 154,09 \mathrm{gr} \\
& \text { Q : } W_{\infty}=2,771 \cdot 10^{-5} \cdot 253,18^{2,915}=
\end{aligned}
$$


De acuerdo con ello las ecuaciones de crecimiento ponderal son:

$$
\begin{aligned}
O^{\prime}: & W_{t}=154,09 \\
& {\left[1-\mathrm{e}^{-0,184(t+2,463)}\right]^{2,912} } \\
O: \quad & W_{t}=281,66 \\
& {\left[1-\mathrm{e}^{-0,193(t+1,670)}\right]^{2,915} }
\end{aligned}
$$

En las tablas I y II se muestran los valores teóricos de los pesos, para cada una de las clases de edad. En la figura 4, se representan gráficamente dichos valores, asi como los pesos medios de cada edad con el in tervalo de confianza del $95 \%$.

Las tasas instantáneas de crecimiento, $G$, obtenidas de la fórmula siguiente:

$$
G=\ln . W_{t+1}-\ln . W_{t},
$$

siendo $\mathrm{W}_{t}$ y $\mathrm{W}_{t+1}$ los pesos a las edades $t$ y $t+1$ respectivamente. (fig. 5 , tabla III)

\section{RETROCALCULO}

Las ecuaciones de relación longitud escama y longitud ejemplar (fig. 2 y 3 ) son:

O $: \mathrm{L}_{n}=17,45+2,63 \cdot \mathrm{R}_{n} \quad(\mathrm{r}=0,937)$

$: L_{n}=26,89+2,40 \cdot R_{n} \quad(r=0,959)$

y las ecuaciones de retrocálculo:

$\sigma^{\prime}: \mathrm{L}_{t}=17,45+\frac{\mathrm{R}_{t}}{\mathrm{R}_{n}} \cdot\left(\mathrm{L}_{n}-17,45\right)$

O : $L_{t}=26,89 \cdots \frac{\mathrm{R}_{t}}{\mathrm{R}_{n}} \cdot\left(\mathrm{L}_{n}-26,89\right)$

En la tabla III se dan las longitudes retrocalculadas para los dos sexos por separado.

\section{CONCLUSIONES}

La determinación de la edad por la lectura de escamas resulta un buen método para

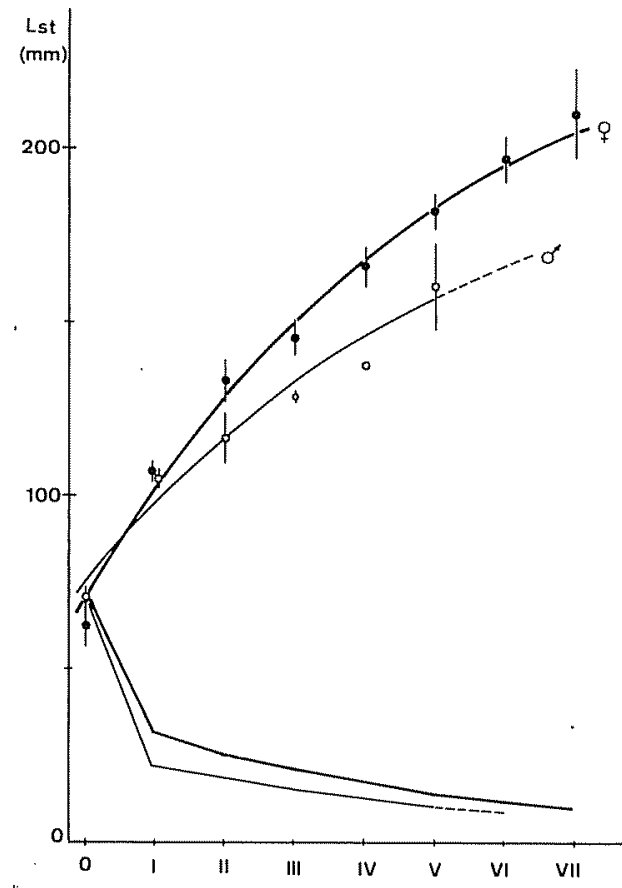

Fig. 4. Representación gráfica de los valores teóricos de peso (modelo VON BIIRTALANFFY (1938)), los valores medios reales y el intervalo de confianza del $95 \%$ de Chondrostoma toxostoma.

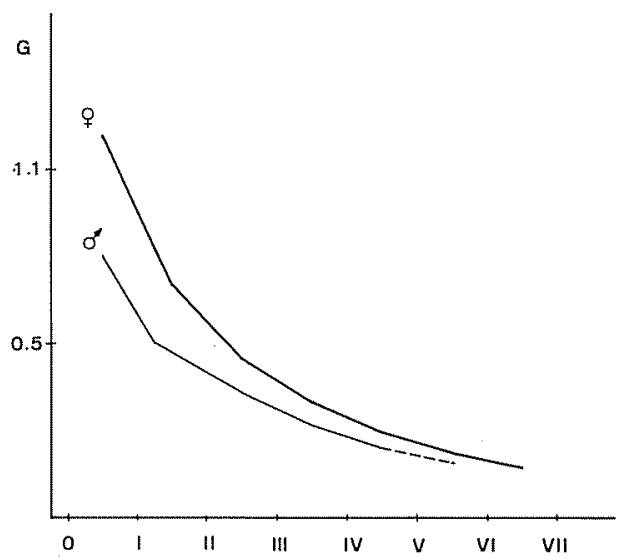

Fig. 5. Tasas instantáncas de crecimiento (G) de Chondrostoma toxostoma. 
TABLA III. Longitudes retrocalculadas (mm) para cada edad de hembras y machos, obtenidas por medición de escamas đe Chondrostoma toxostoma. Vallot, 1837.

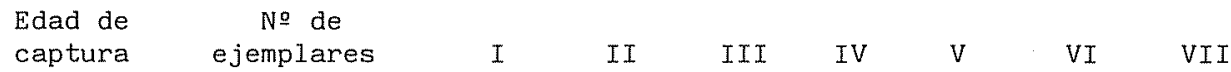

$\begin{array}{lr} & \\ \text { I } & \\ \text { II } & 3 \\ \text { III } & 6 \\ \text { IV } & 7 \\ \text { V } & 12 \\ \text { VI } & 6 \\ \text { VII } & 5\end{array}$

HE M B R A S

100,27

$110,87123,87$

$101,86119,87 \quad 132,20$

$98,43117,37131,08141,56$

$96,56 \quad 117,91 \quad 132,19 \quad 145,18 \quad 158,39$

$98,73 \quad 122,49 \quad 137,25 \quad 149,65 \quad 162,76 \quad 175,47$

$100,37124,54142,28 \quad 156,02 \quad 170,30183,11 \quad 195,35$

Medias

$101,01 \quad 121,01 \quad 135,02 \quad 148,12 \quad 163,82 \quad 179,29195,35$

$M A C H O S$

I $\quad 18 \quad 87,48$

II $\quad 7 \quad 79,29109,80$

III $\quad 7 \quad 78,94104,32121,91$

IV $\quad 1 \quad 88,61105,69119,92128,46$

V 3

Medias

$90,02 \quad 108,00128,33142,84 \quad 153,19$

$84,87106,95123,39135,65153,19$

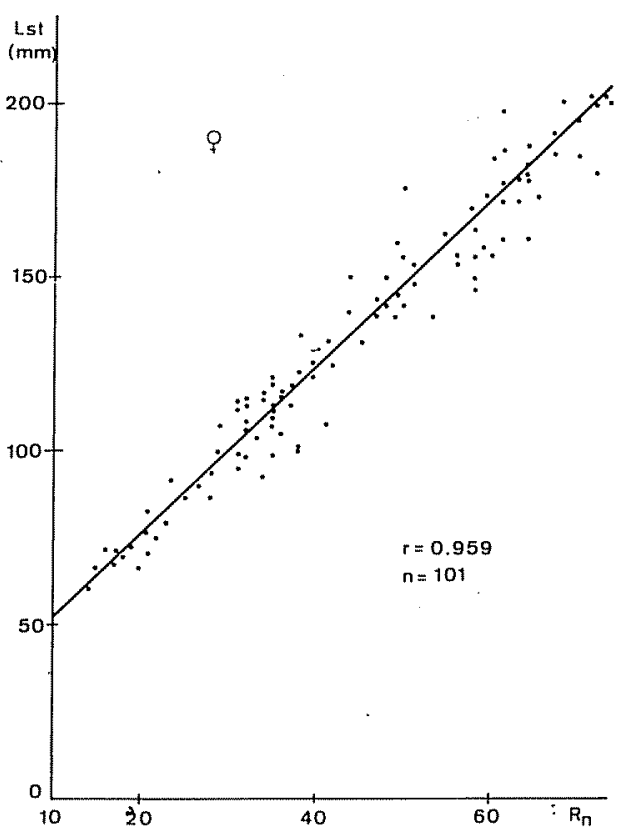

Fjg. 6. Relación entre la longitud standard (Lst, en $\mathrm{mm}$ ) y el radio posterior de la escama ( $\mathrm{Rn}$, en $\mathrm{mm}$ $x$ 10) de los machos de Chondrostoma toxostoma.

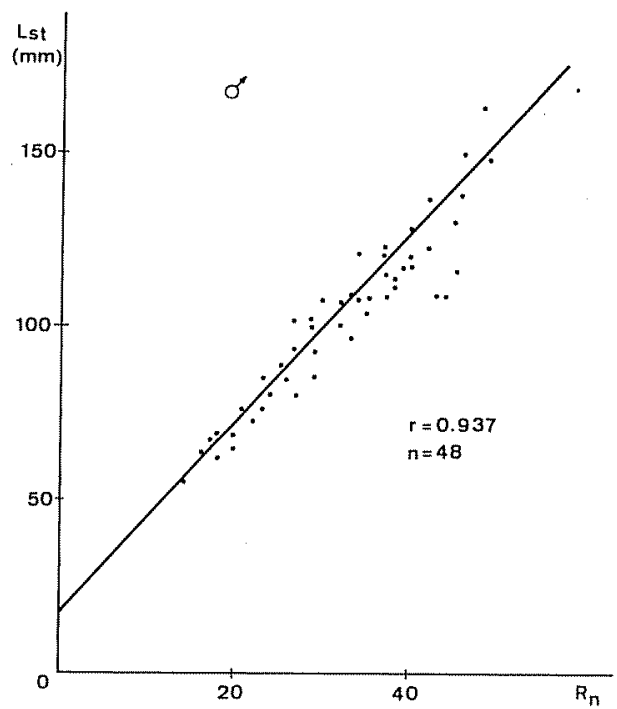

Fig. 7. Relación entre la longitud standard (Lst, en $\mathrm{mm}$ ) y el radio posterior de la escama ( $\mathrm{Rn}$, en $\mathrm{mm}$ $x$ 10) de las hembras de Chondrostoma toxostoma. 
la especie Chondrostoma toxostoma. La formación de los anillos (annuli) se realizan en los meses de mayo y junio. Las clases de edad más grandes encontradas son la "VII" en hembras y la " $\mathrm{V}$ " en los machos. La ausericia de otras clases de edad superiores en ambos sexos, se interpreta como una consecuencia de hallarse la especie en el límite de sus posibilidades de colonización por causa de la salinidad.

Las longitudes máximas encontradas son
$167 \mathrm{~mm}$. para los machos y $222 \mathrm{~mm}$. para las hembras con una relación longitud-peso más exponencial en las hembras que en los machos (fig. 1). Esto es común a diversas especies de Ciprínidos.

Las tendencias de las curvas de crecimiento lineal y ponderal son similares en ambos sexos, aunque el crecimiento es más rápido y continuado en el segundo caso. Las hembras experimentan un mayor crecimiento que los machos, especialmente en peso.

\section{BIBLIOGRAFIA}

BAGINAAL, T.B. (ed.) 1974. Ageing of Fish. Unwin Bros. Ltd., Old Woking, 234 págs.

BAGENAL, T.B. \& F.W. TESCH. 1978. Age and Growth. In: Methods for Assessment of Fish Production in Fresh Waters. (BAGENAL, T.B. ed.): págs. 101-130. I.B.P. Handbook 3, Blackwell. Oxford.

CHUGUNOVA, N.I. 1963. Age and growth studies in fish. Jerusalem, 132 págs. (Translation of CHUGUNOVA, N.I. 1959. Rukovodstvo po izucheniy'a yozrasta ry $b$. Moscow).

DIMESTRF, M.: ROIG, A.; SOSTOA, A. \& SOSTOA, F.J. 1977. Contribució a l'estudi de l'ictiofauna continental del delta de l'Tbre. Treb. Inst. Cat. Hist. Nat., 8: 145-226.

DICKIE, L.M. 1978. Mathematical models of growth. In: Methods for Assessnent of Fish Production in Fresh Waters. (BAGENAL, T.B. ed.): 130-136. I.B.P. Handbook 3, Blackwell. Oxford.

DUNCAN, K.W. 1980 . On the back-calculation of fish lengths; modifications and extensions to the Fraser-Lee equation. J. Fish Biol., 16: 725730.

FRASER, C.M. 1916. Grow th of the spring salmon. Trans. Pacific Fish Soc., Seattle, Second Ann. Meeting, 1915: 29-35.

FORD, I: 1933. An account of the herring investigations conducted at Plymouth during the years from 1924-1933. J. mar. biol. Assoc. U. K., 19: 305-384.

HOCHMAN, L. \& JIRASI:K, J. 1969. Fish Stocking in Upper part of the Oslava Drainage Area and Grow th of Main Fish Species Prior to Construction of the Dammed Lake at Mostisté. Acta Univ. agricult. Bmo, 17 (2): 415-423.
HOFSTEDE, A.E. 1970. Scale Reading and Backcalculation (Roach and Dace of known age). Documtie Rapp. Afdeling Sportwisserij en Bereopsbinnenvisserij, 11: 15 págs.

KLIMCZYK-JANIKOWSKA, M. 1973. Cyprinid (Chondrostoma nasus L.) from the River Raba. Acta Hydrohiol. Krakon', 15 (2). 197-213.

LEE, R.M. 1920. A review of the methods of age and growth determination in fishes by means of scales. Fishery Invest., Lond. Ser. 2, 4: 1-32.

LOBON-CERVIA, J. \& ELVIRA, B. (en prensa). On some aspects of the biology of the Iberian Nase (Chondrostoma polylepis polylepis, Steind., 1865) in Pinilla reservoir (River Lozoya). 1 Jomadas Ictiologia Ibérica, León. 1981.

LOSOS, B.; PEÑAZ, M. \& KUBICKOVA, J. 1980. Food and grow th of fishes of the Jihlava River. Acta Sc. Nat. Bmo., 14 (1): 146.

LUDINI, G. \& NARDI, PA. 1979. Osservacioni sull'ecologia di una popolazione de Chondrostoma soetta $\mathrm{Bp}$., (Cyprinidae, Osteichthyes, Pisces) del fiume Ticino. Quad. del Sile e altri fiumi, Ril. ital. Potamol., 2-3: 48-60.

PRAWOCHENSKF, R. 1963. The Age and Growth Rates of the Nose Carp-Chondrostoma nasus (L.) from S.W. part of Poland. Rockniki Nauk Roln., 53 (1): 161-182.

VELEDAR, I: \& KOSORIC, DJ, 1974. The methods for age determination of Chondrostoma phoxinus Heckel, 1843 from the waters of Livan jsko polje. Ichth yologia, 6 (1): 93-99.

VON BERTALANFFY, L. 1938. A quantitative theory of organic growth. Human. Biol., 10: 181-243.

WALFORD, L.A. 1946. A new graphic method of describing the growth of animals. Biol. Bull. mar: biol. lab. Woods Hole, 90: 141-147. 